\title{
Segmentation of coronal holes in solar disk images with a convolutional neural network
}

\author{
Egor A. Illarionov, ${ }^{1,3 \star}$ Andrey G. Tlatov, ${ }^{2,3}$ \\ ${ }^{1}$ Moscow State University, Moscow, 119991, Russia \\ 2 Kislovodsk Mountain Astronomical Station of the Pulkovo Observatory, Kislovodsk, 357700, Russia \\ 3 Kalmyk State University, Elista, 358000, Russia
}

Accepted XXX. Received YYY; in original form ZZZ

\begin{abstract}
Current coronal holes segmentation methods typically rely on image thresholding and require non-trivial image pre- and post-processing. We have trained a neural network that accurately isolates CHs from SDO/AIA $193 \AA$ solar disk images without additional complicated steps. We compare results with publicly available catalogues of CHs and demonstrate stability of the neural network approach. In our opinion, this approach can outperform hand-engineered solar image analysis and will have a wide application to solar data. In particular, we investigate long-term variations of $\mathrm{CH}$ indices within the solar cycle 24 and observe increasing of $\mathrm{CH}$ areas in about three times from minimal values in the maximum of the solar cycle to maximal values during the declining phase of the solar cycle.
\end{abstract}

Key words: Sun: corona - solar wind - techniques: image processing - methods: data analysis

\section{INTRODUCTION}

Coronal holes (CHs) are regions of open magnetic fields in the solar corona. They are typically associated with high speed solar wind streams (e.g. Altschuler et al. 1972; Krieger et al. 1973) and provide an estimation of solar wind parameters and corresponding geomagnetic effects (e.g. Nolte et al. 1976; Robbins et al. 2006; Obridko et al. 2009; Abramenko et al. 2009; Rotter et al. 2015). An accurate $\mathrm{CHs}$ segmentation procedure is essential for more detailed investigation of this relationship and implementation of space weather forecasting routines.

CHs are best seen by eye in images in the $193 \AA$ wavelength. They appear as dark regions due to their lower density and temperature in contrast to surrounding atmosphere (see Priest 2014). CHs have irregular profile and may occupy more than $10 \%$ of visible solar hemisphere.

For segmentation of $\mathrm{CHs}$ one typically applies rulebased approaches that include image thresholding and region growth steps. For example, Krista \& Gallagher (2009) identify CHs using a histogram-based intensity thresholding. The Automatic Solar Synoptic Analyzer (ASSA; Hong et al. 2014) also includes thresholding at some fraction of median pixel value. Morphological image analysis, thresholding and smoothing was applied by Henney \& Harvey (2005) for $\mathrm{CH}$ detection in solar spectroheliograms. Scholl \& Habbal

^ E-mail: egor.mypost@gmail.com
(2008) apply contrast enhancement in a multi-passband detection method in $171 \AA, 195 \AA$, and $304 \AA$ passbands. Similarly, multi-thermal intensity cut is a key step in the Coronal Hole Identification via Multi-thermal Emission Recognition Algorithm (CHIMERA; Garton et al. 2018). The Spatial Possibilistic Clustering Algorithm (SPOCA) requires a proper intensity normalization to apply fuzzy clustering method for image segmentation (Barra, V. et al. 2009; Verbeeck, C. et al. 2014).

While contrast enhancement, thresholding and geometrical considerations were massively exploited in computer vision for may years in a number of domains, more and more recent publications demonstrate that computer vision algorithms based on neural networks can provide significantly better results in object detection in comparison to conventional hand-engineered algorithms (see e.g. Chen et al. (2014) for a review). In application to solar data, Díaz Baso \& Asensio Ramos (2018) presented deep learning techniques for enhancing SDO/HMI images, while Asensio Ramos et al. (2017) trained a neural network to estimate horizontal velocities at the solar surface. We failed to find any publication relevant to neural networks usage for active regions segmentation, although the amount of available data for neural network training is more than enough.

In our opinion, investigation of neural networks with respect to segmentation of solar active regions allows some possibilities beyond the capacity of hand-engineered algorithms. The point is that neural networks can learn a 
methodology of active regions isolation that was used e.g. at some observatory or by particular astronomer. Applying the trained network to a larger time interval one can create an extended dataset of active regions isolated by methodology similar to original one. This trick can hardly be done by manual threshold levels adjustment since many aspects of individual image perception are difficult to formalize explicitly. Such an extended dataset would be interesting in respect to e.g. calibration of sunspot number series (see Clette et al. (2014) for review of the problem).

This paper is aimed to present an approach based on convolutional neural networks (CNNs) for segmentation of coronal holes in solar disk images and attract interest in application of this technique to wider scope of astrophysical problems. We demonstrate that $\mathrm{CNN}$ can outperform hand-engineered methods of $\mathrm{CH}$ detection and investigate properties of obtained CHs within the solar cycle 24 .

\section{CONVOLUTIONAL NEURAL NETWORKS FOR IMAGE SEGMENTATION}

Convolutional neural network consists of an input and output layers and a number of hidden layers. Hidden layers can be represented by convolutional, resizing, normalization and some other type of layers. The detailed definition of layers and an intuition behind them can be found e.g. in Bishop (1995), Deng \& Yu (2014) or Goodfellow et al. (2016). The way layers are connected defines a neural network architecture. Training of neural networks consists in optimization of trainable variables with respect to loss function between target values and output predictions of the model.

\subsection{Network architecture}

For segmentation of $\mathrm{CHs}$ we implemented an architecture similar to the U-Net architecture, elaborated for biomedical image segmentation (see Ronneberger et al. 2015). It is worth noting that U-Net-like networks demonstrate superior results in very different segmentation problems, in $2 \mathrm{D}$ or 3D. For example, in satellite image analysis (Iglovikov et al. 2017a) or medical image analysis (Iglovikov et al. 2017b; Ching et al. 2017; Çiçek et al. 2016). These facts inspired us to apply U-Net-like network for segmentation of CHs.

Fig. 1 shows an architecture of the neural network that we suggest for CHs segmentation. For simplicity we will also call this network U-Net. The network input and output layers have the same shape $256 \times 256 \times 1$. Input layer accepts grayscale image of solar disk resized to $256 \times 256$ pixels. Output layer produces a predicted segmentation map, i.e. an image of the same shape $256 \times 256$, where pixels assigned to $\mathrm{CHs}$ have values close to 1 , while other pixels have values close to 0 . Hidden layers are represented by operations named ConvBlock, MaxPooling, TransposedConv, Concatenation and Conv and are explained below.

ConvBlock is a compact notation of two successive convolutional layers with activation function called "exponential linear unit" (ELU, see Clevert et al. 2015). Both convolutional layers have the same number of filters, these numbers are shown in Fig. 1. Note that we double number of filters in ConvBlocks from top to bottom of the network starting from 24 . Kernel size for convolutions is $3 \times 3$ (any other convolutions in our network also have kernel size $3 \times 3$ ). Spatial resolution remains unchanged after convolutions due to zero padding and unitary stride. In the end of ConvBlock we apply a dropout layer (Srivastava et al. 2014) with rate 0.1. The dropout layer deactivates a certain set of neurons chosen at random with given rate during training phase. This helps the neural network to improve over-fit.

MaxPooling layer reduces spatial resolution to a half using maximum function to summarize subregions. Channel dimension remains unchanged. Size of the pooling window is 2 for both spatial axes, strides are also equal to 2 .

TransposedConv is a convolutional layer with transposed gradient (see Dumoulin \& Visin 2016) and ELU activation function. Due to strides equal to 2 for all spatial axes, the output spatial dimensions are doubled. Sometimes this operation is called "deconvolution". Here we also apply dropout with rate 0.1 .

Concatenation operation simply stacks two tensors along channel axis.

Conv layer is a convolution layer with sigmoid activation function $\sigma(x)=\left(1+e^{-x}\right)^{-1}$. Sigmoid function ensures that the network output values are in $[0,1]$ range. Recall that we interpret these values as probability of pixels to belong to $\mathrm{CH}$.

Intuition behind the U-net architecture can be understood as follows. Left branch of the network compress an input image to extract context information. Connections between left and right branches pass context information to improve its localization during decompression.

\subsection{Dataset}

A dataset for $\mathrm{CH}$ segmentation consists of pairs of solar disk images and corresponding segmentation maps. We exploit one image per day in $1024 \times 1014$ resolution from the Solar Dynamic Observatory (SDO) Atmospheric Imaging Assembly (AIA; Lemen et al. 2012) $193 \AA$ catalogue. It covers time period from 2010 to the present time. The catalogue is accessible e.g. at the Joint Science Operation Center (JSOC; http://jsoc.stanford.edu/). Segmentation maps are obtained from data archive of the Kislovodsk Mountain Astronomical Station that is available and daily updated at http://observethesun.com. Recall a $\mathrm{CH}$ processing algorithm for this data archive (we will refer to it as the region growth algorithm).

$\mathrm{CHs}$ are obtained as a result of semi-automatic procedure of processing SDO/AIA $193 \AA$ solar disk images. This procedure inherits the ones applied in Tlatov et al. (2014) for CHs identification from observations in the He I $10830 \AA$ line made at Kitt Peak Observatory (from 1975 to 2003) and in the EUV $195 \AA$ wavelength with SOHO/EIT (from 1996 to 2012) with some improvements.

In the first step, the algorithm detects solar disk centre and radius. Then, it computes pixels intensity distribution within the solar disk and selects initial regions with pixel intensity below 0.4 quantile. Here it also detects the quite Sun level $(L Q S)$ as the mode of the distribution. Note that the threshold parameter for initial regions, which is 0.4 by default, is controlled by the observatory data engineer and can be varied in order to achieve better agreement with visual expectation. The next step consists of iterative region 


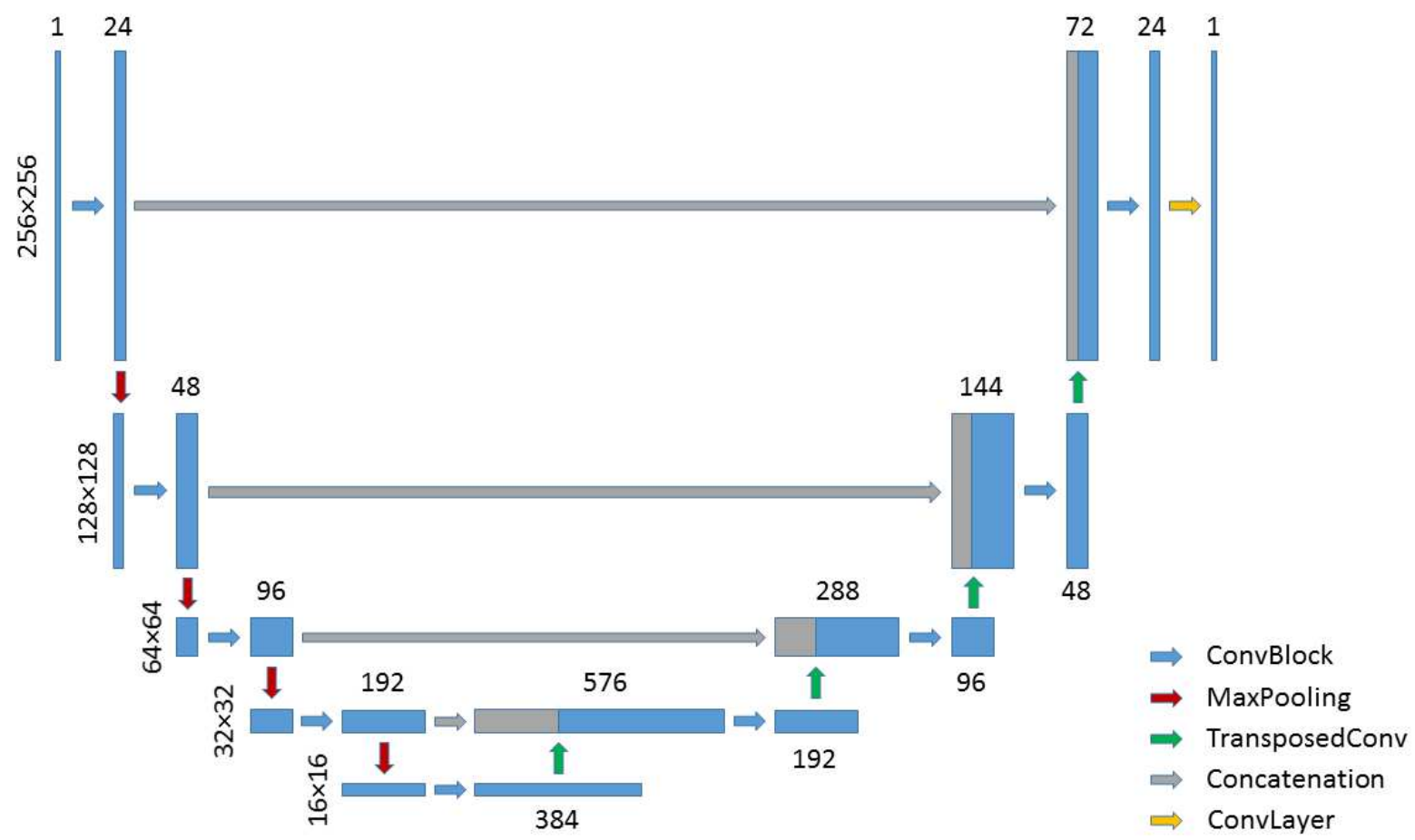

Figure 1. Neural network architecture for CHs segmentation. Each box corresponds to a multi-channel feature map. Width of boxes and numbers above or below boxes represent its channel dimension, while height of each box and numbers to the left of boxes represent its spatial dimensions. Color arrows correspond to different operations annotated in the figure.

growth from initial regions. At each iteration new pixels with intensity above $0.4+0.05 * i * L Q S$ are merged into region, where $i$ is the current iteration step. Iteration stops when the region area growth rate rapidly leaps up. At this moment the region starts to occupy regions of the quite Sun.

Obtained regions with areas less than $2 \mathrm{~K}$ millionth of a solar hemisphere (MSH) are filtered out. Finally, $\mathrm{CH}$ candidate regions are inspected visually and the threshold parameter for initial regions is manually corrected if necessary. The result containing coordinates of $\mathrm{CHs}$ boundaries and parameters of CHs (e.g. area and elongation) is added to the map of solar active regions at http://observethesun.com.

Totally the dataset contains 2916 pairs of solar disk images and corresponding segmentation maps. We divide it into train and test parts, where train part contains all pairs before 2017 (2385 items) and test part contains all pairs starting from 2017 (513 items). Note that we do not mix dates in train and test subsets. The point is that otherwise the relative low day to day variability of $\mathrm{CHs}$ (see e.g. Zhang et al. 2003) will result in very similar items in the test and train subsets and thus will distort the model evaluation.

\subsection{Data preprocessing}

Image preprocessing consists of resizing of original $1024 \times$ 1024 images to shape $256 \times 256$, rescaling of pixel values to $[0,1]$ range from original $0,1 \ldots 255$ intensity values, rotation of each image at random angle in 0, 90, 180, 270 degrees and reversing along each spatial axis with probability 0.5 . Random transformations included in the preprocessing increase the amount of training data (augment the dataset) and reduce over-fit (see e.g. Perez \& Wang 2017).

Segmentation maps are given by images of shape $256 \times$ 256 resized from original $1024 \times 1024$ maps. Each pixel is equal to 1 or 0 depending on whether it belongs to $\mathrm{CH}$ or not.

\subsection{Training}

We use preprocessed solar disk images and corresponding segmentation maps to train the network with the adaptive moment estimation optimization algorithm(Adam; Kingma \& Ba 2014) with learning rate 0.001.

Similarity measure between predicted and target segmentation map (loss function for optimization algorithm) is computed by a pixel-wise binary cross-entropy defined as

$\operatorname{loss}(Y, \hat{Y})=-\frac{1}{n^{2}} \sum_{i, j=0}^{n} y_{i j} \log \hat{y}_{i j}+\left(1-y_{i j}\right) \log \left(1-\hat{y}_{i j}\right)$,

where $Y=\left(y_{i j}\right), \hat{Y}=\left(\hat{y}_{i j}\right)$ are target and predicted segmentation maps correspondingly.

Training procedure consists of iterations over the dataset with subsets of certain size and updating trainable variable of the network after each iteration according to given optimization algorithm. The subsets are called batches, one iteration over the whole dataset is called epoch. Once the dataset is exhausted, we permute its items at random and start a new epoch. Thus we obtain batches composed of random items and improve convergence of the optimization algorithm (see e.g. Meng et al. 2017). As it was 


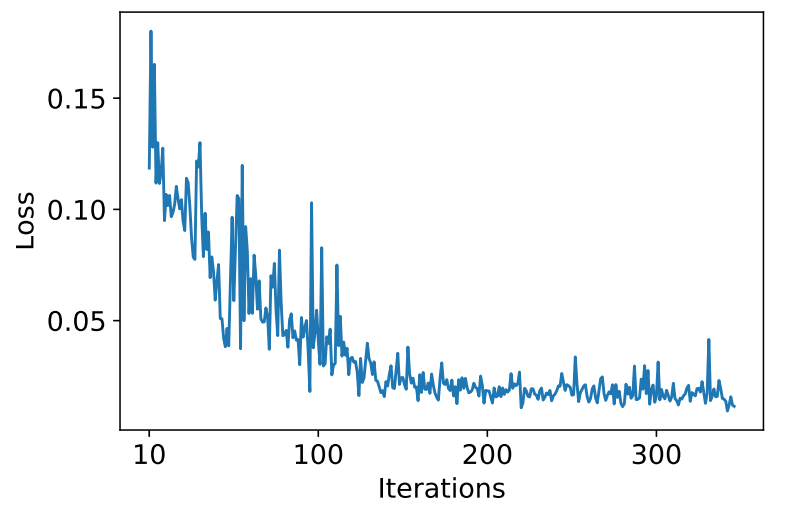

Figure 2. Loss function (binary cross-entropy) against iterations. First ten iterations are hidden due to large values of the loss function.

shown e.g. in Radiuk (2017), larger batch sizes provide better classification or segmentation results for neural networks. However, there is always a trade-off between batch size, neural network size and and CPU/GPU memory limits. The UNet network has about $6.2 \mathrm{M}$ of trainable variables. We used the GeForce GTX 1060 graphics card with 6 Gb memory for experiments and batch size 20. Training time is about 15 minutes using. It is worth noting that the same experiments on CPU were more than 10 times as long for us.

Fig. 2 shows how the loss function varies with iterations. We observe that 3 epochs (357 iterations) are enough to reach a plateau in the loss function, which means a convergence to some proper local minimum. Averaged loss at the final epochs is equal to 0.018 .

The source code necessary to reproduce the training procedure is provided in a public repository https://github.com/observethesun/coronal_holes. Python and TensorFlow are required to run the code.

\section{RESULTS}

The trained network was used to predict $\mathrm{CHs}$ in the test part of the dataset. Recall that the test part consists of daily SDO/AIA $193 \AA$ images from January 2017 till June 2018. The Fig. 3 shows an example of $\mathrm{CHs}$ segmentation (that day, January 30, 2017, U-Net found the largest $\mathrm{CH}$ in the test period, about $13 \%$ of the solar hemisphere).

We do not apply any post-processing for obtained segmentation maps due to the following reasons. First, we want to demonstrate a baseline for $\mathrm{CHs}$ segmentation with neural network, i.e. a quality that can be expected from the neural network trained just on original images and target segmentation maps. Second, we believe that most of drawbacks in predicted outputs can be improved not due to handengineered corrections, but due to improvements in the network architecture or in the network training scheme. Third, in our opinion, comparison of CHs against magnetograms and discarding of some $\mathrm{CHs}$ with respect to their unipolarity is a very speculative procedure and requires a detailed investigation, which is out of the scope of this paper.

We appreciate that $\mathrm{CHs}$ isolated with the above mentioned procedure are rather only candidates to $\mathrm{CHs}$. How-

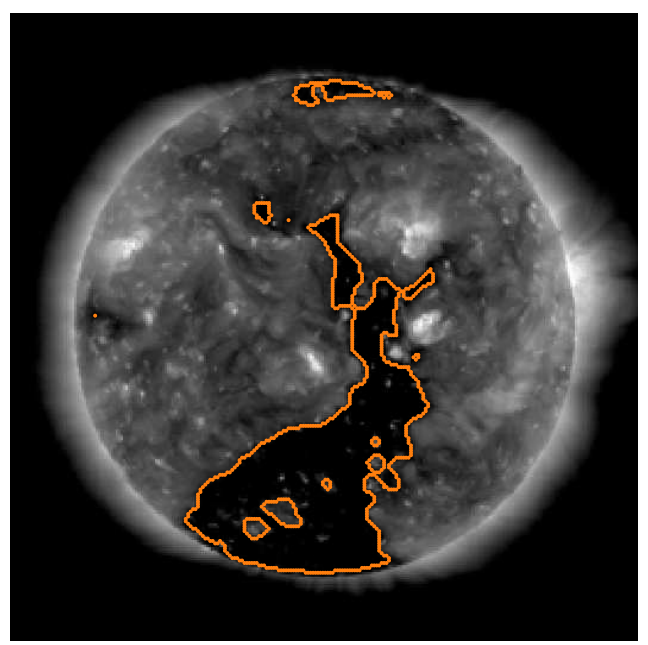

Figure 3. Contours of coronal holes isolated by U-Net (January 30,2017$)$. Here and below image size is $256 \times 256$ pixels.

ever, since the ground truth is unknown, we will concentrate on demonstration of stability of the procedure and its comparison with alternative approaches. For comparison we consider catalogue of $\mathrm{CH}$ isolated by CHIMERA (available at https://solarmonitor.org/data/, by SPOCA (available at http://lmsal.com/isolsearch and via the Application Programming Interface (API) of the Heliophysics Events Knowledgebase (HEK)) and $\mathrm{CH}$ maps from test part of our dataset. We failed to find any other public available longterm catalogue of isolated and digitized CHs boundaries and its parameters. It would be fruitful to include into investigation $\mathrm{CH}$ maps from the National Oceanic and Atmospheric Administration (NOAA) Space Weather Prediction Center (SWPC) and CH maps from Automatic Solar Synoptic Analyzer (ASSA). However, the first ones are not digitalized (i.e. $\mathrm{CHs}$ boundaries are not extracted), the second ones include only latest map.

\subsection{Dice score as a similarity measure}

We will compare $\mathrm{CH}$ segmentation maps using the Dice similarity coefficient (Dice 1945). For two sets $A$ and $B$ it is expressed as

$\operatorname{dice}(A, B)=\frac{2|A \cap B|}{|A|+|B|}$,

where $|A|$ is the cardinal of set $A$. Intuitively, this can be seen as a percentage of overlap between the two sets.

First, we compare $\mathrm{CH}$ segmentation maps predicted with U-net with $\mathrm{CH}$ maps for the same day obtained with the region growth algorithm. Fig. 4 shows the dice score between two segmentation maps for the test time period.

We found the dice score fluctuates moderately near its mean values which is equal to 0.81 with standard deviation equal to 0.1 . To better understand this value it can be noted 


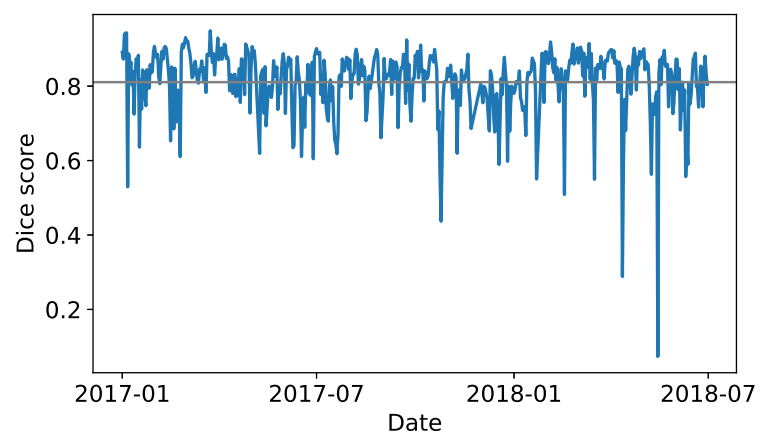

Figure 4. Dice scores between segmentation maps predicted with U-net and obtained with the region growth algorithm for the test time period. Horizontal line shows mean value 0.81 .

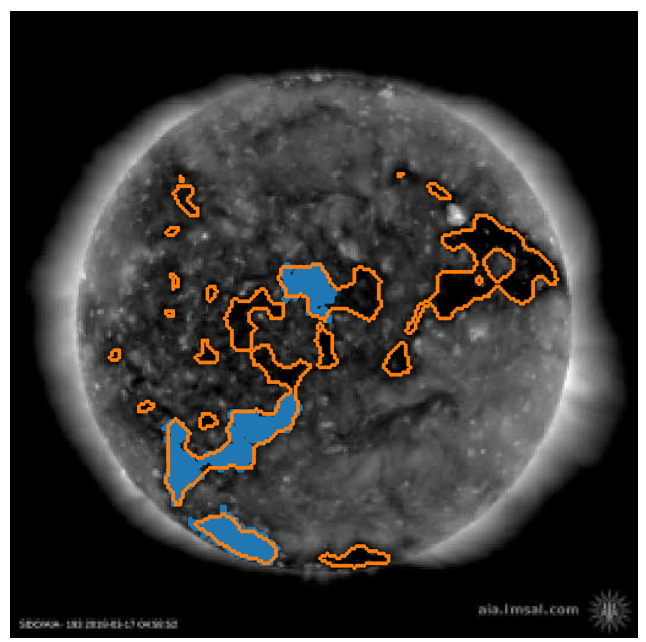

Figure 5. Substantial difference between $\mathrm{CH}$ s isolated by the region growth algorithm (blue regions) and by U-net (orange contours). March 17, 2017. Dice score is 0.55.

that two concentric circles with outer radius $20 \%$ greater than inner have the dice score 0.82 .

Visual comparison of predicted and target segmentation maps brings us to conclusion that U-Net finds more regions related to $\mathrm{CHs}$ and gives more accurate approximation of them. Rare outliers in Fig 4 are mostly caused by substantial underestimating of $\mathrm{CH}$ regions by the region growth algorithm. An example of the outlier with dice 0.55 is shown in Fig. 5. Again, we observe that U-Net provides better segmentation result.

Dice score also can be used to validate a stability of segmentation maps. Indeed, considering $\mathrm{CH}$ segmentation maps not in the solar disk, but in the Carrington coordinate system, we can naturally expect that images do not vary a lot from day to day. Thus, it makes sense to compute the dice score. To neglect the rotation effects we consider the dice score between current map and union of maps for

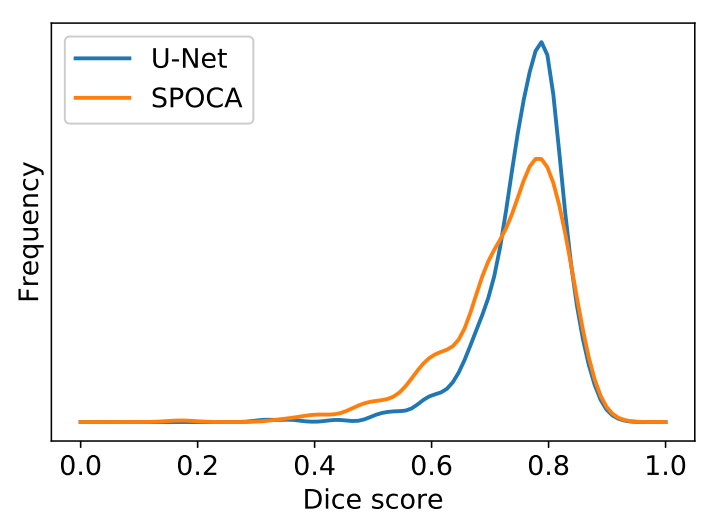

Figure 6. Distribution of dice scores for segmentation maps produced by U-Net (blue line) and SPOCA (orange line).

the day before and after the current day. Then we compute a histogram of dice scores obtained for the test time period. Fig. 6 shows the smoothed histograms for U-Net and SPOCA.

One can note in the Fig. 6 that while modes of the distributions are very close, frequency for U-Net is about $25 \%$ higher. This means, that U-Net produces more consistent segmentation maps and demonstrates better stability as compared to SPOCA. Unfortunately, catalogue of $\mathrm{CH}$ isolated by CHIMERA does not contain boundaries of $\mathrm{CH}$ and thus is not included into this analysis.

\subsection{Time-variation of the $\mathrm{CHs}$ areas}

Here we consider another aspect of stability of CHs segmentation algorithm, which is a time-variation of total CHs area. Since typical CHs exist more than one solar rotation period, it is highly unlikely to observe rapid day-to-day leaps in a graph of CHs area.

Recall that one can calculate areas of solar active regions in sky plane and measure them in percentage of solar disk or, alternatively, estimate reprojected areas and measure them in percentage of solar hemisphere. In practice, however, estimation of reprojected areas can be inaccurate since many $\mathrm{CHs}$ are located near poles where projection effects are substantial. On the other hand, ares measured in sky plane can also be misleading since two $\mathrm{CHs}$ of the same area (in sky plane) located near disk centre and near pole have very different actual (reprojected) areas. Below we will show areas calculated in both ways.

Fig. 7 (upper panel) shows a daily variation of total $\mathrm{CHs}$ area measured in sky plane. We observe that U-Net and CHIMERA give very similar results with a correlation coefficient equal to 0.76. At the same time, total $\mathrm{CHs}$ areas according to SPOCA are up to 3 times fewer in contrast to CHIMERA and U-Net. We selected a day (March 19, 2018) when the difference is most prominent and show isolated CHs corresponding to each algorithm in Fig. 8. In our opinion, the map B (SPOCA) clearly underestimates $\mathrm{CHs}$ regions, the map $\mathrm{C}$ (CHIMERA) somehow overestimates $\mathrm{CHs}$ with respect to the $193 \AA$ image, while the map A (U-Net) produces the most accurate segmentation that corresponds to visual expectation. Note that total $\mathrm{CHs}$ area shown in 

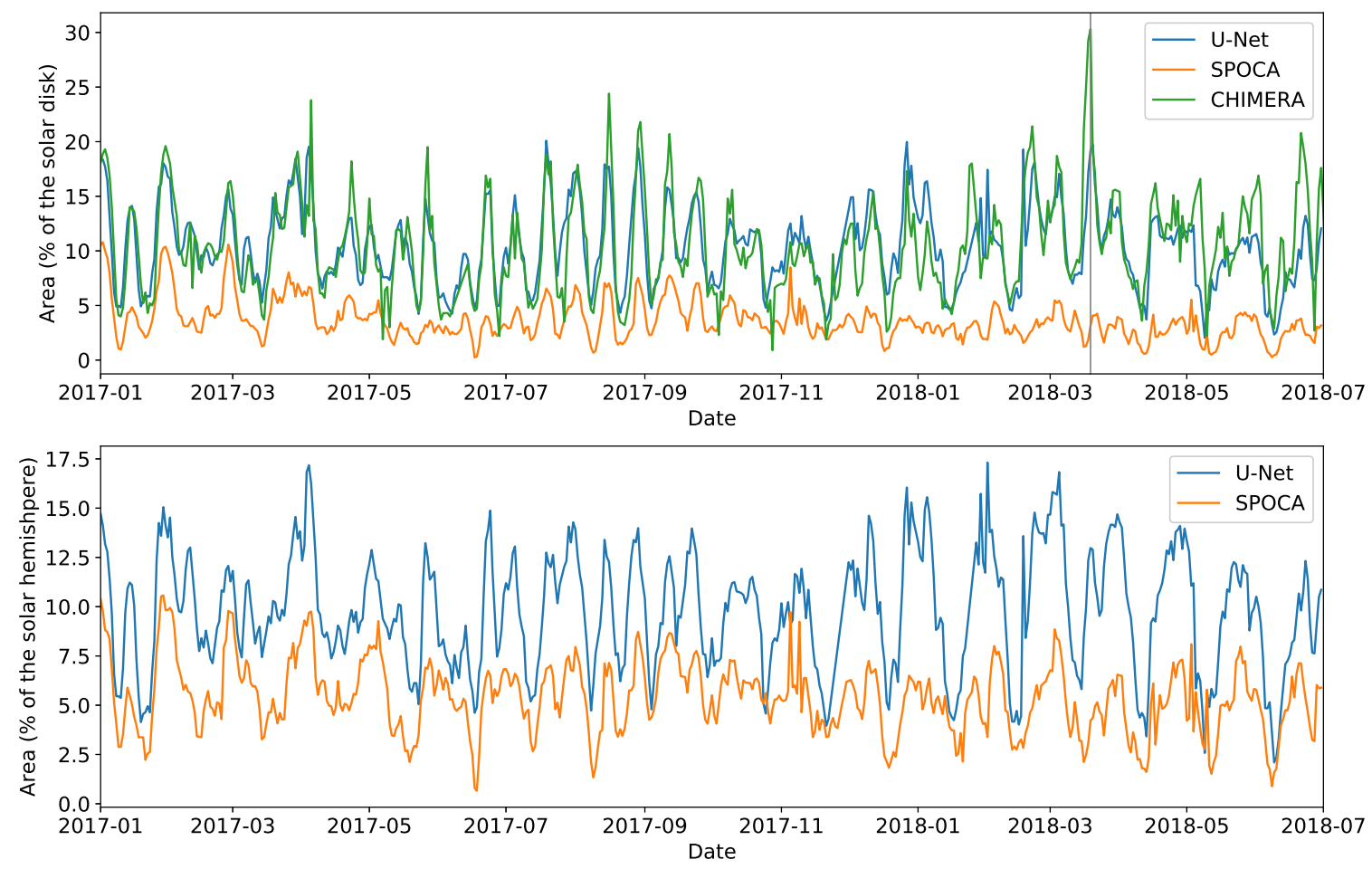

Figure 7. Upper panel: total area of coronal holes in plane of sky. Blue line is for U-Net, orange line if for SPOCA, green line is for CHIMERA algorithm of CHs segmentation. Vertical line shows a day selected for detailed comparison (see Fig. 8). Lower panel: total reprojected area of coronal holes. Blue line is for U-Net, orange line if for SPOCA algorithm of CHs segmentation.

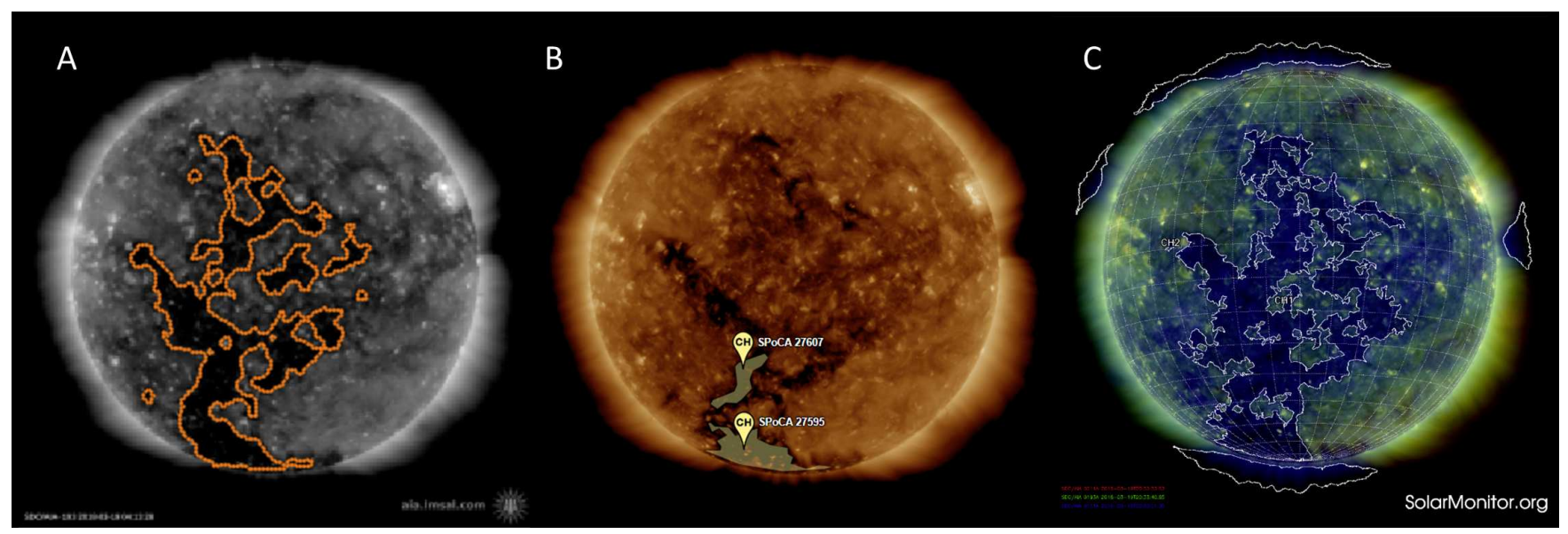

Figure 8. Segmentation maps for March 19, 2018 by U-Net (A), SPOCA (B) and CHIMERA (C).

Fig. 8 is $19 \%$ of the solar disk according to U-Net, $3 \%$ for SPOCA and $30 \%$ for CHIMERA.

In Fig. 7 (lower panel) we show a daily variation of total reprojected $\mathrm{CHs}$ area. It does not include corresponding plot by CHIMERA since we observe that almost all reprojected areas in catalogue of CHs isolated by CHIMERA are in order of magnitude larger than that for SCOCA or
U-Net. The point is that according to the source code at https://github.com/TCDSolar/CHIMERA CHIMERA estimates reprojected area relative to position of $\mathrm{CHs}$ centroid. In our opinion, this method can make sense for small objects, i.e. pores, while for large object, i.e. $\mathrm{CH}$, the more accurate estimation requires e.g. reprojection of each pixel within the CH. For example, consider a day March 9, 2018. 


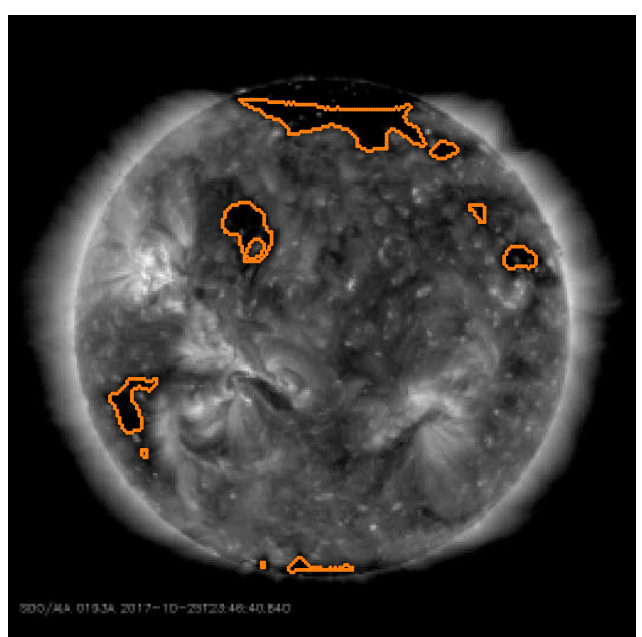

Figure 9. Almost absent solar limb can result in incorrect $\mathrm{CH}$ segmentation (see the $\mathrm{CH}$ near the north pole).

According to CHIMERA, the largest $\mathrm{CH}$ near the north pole occupies $2.7 \%$ of the solar disk area and has reprojected area $1.3 \cdot 10^{6} \mathrm{Mm}^{2}$. However, assuming solar radius equal to $6.95 \cdot 10^{2} \mathrm{Mm}$ we obtain that this $\mathrm{CH}$ should cover $1.3 \cdot 10^{6} / 2 \pi\left(6.96 \cdot 10^{2}\right)^{2} \cdot 100 \%=42.7 \%$ of the solar hemisphere, which does not correlate with visual expectation and is a clear overestimation. For comparison, corresponding $\mathrm{CH}$ isolated by U-Net has an area $3.5 \%$ of the solar disk and $4.6 \%$ of the solar hemisphere.

Considering Fig. 7 we observe that areas by SPOCA are about half of areas by U-Net, but both demonstrate similar variations with correlation coefficient 0.66.

\subsection{Difficult cases}

We have noted several occasional cases, when U-Net predicts unnatural CHs. They occurs when the solar limb separating $\mathrm{CH}$ and the outer space is almost absent, e.g. as one near the north pole shown in the Fig. 9 for October 25, 2017.

The point is that the extremely thin solar limb can not propagate deeper into U-Net layers and fire neurons responsible for $\mathrm{CH}$ detection. However, one can easily improve the situation just by a prior segmentation of the solar disk. In the Fig. 10 we set maximal intensity to pixels outside the solar disk. As we can see, now U-Net isolates $\mathrm{CH}$ near the north pole correctly.

Thus, to avoid the limb effects we recommend including a segmentation of the solar disk in image preprocessing pipeline.

\subsection{CHs in the solar cycle 24}

Here we present a variation of $\mathrm{CH}$ areas from the beginning of SDO/AIA observations in 2010 up to the present time according to U-Net segmentation. Note that this

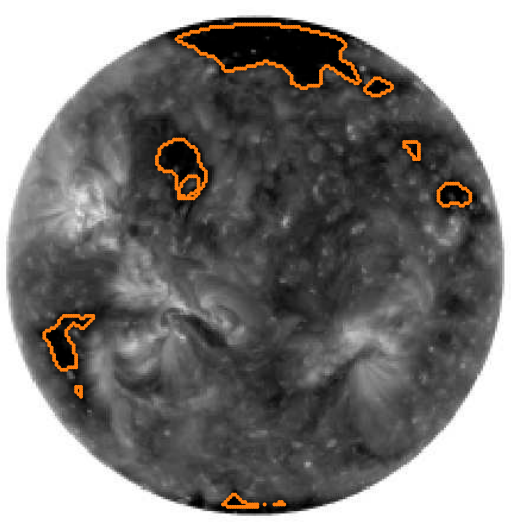

Figure 10. Prior setting the maximal intensity to pixels outside the solar disk improves the $\mathrm{CH}$ segmentation near the solar limb in comparison to the Fig. 9

time period includes both train and test periods for UNet and covers approximately the solar cycle 24. For comparison we consider a variation of the solar wind (SW) speed measured onboard the Advanced Composition Explorer (ACE Stone et al. 1998). Daily averaged SW speeds were obtained from level $2 \mathrm{ACE}$ data at http://www.srl.caltech.edu/ACE/ASC/level2/.

Due to the fact that visible $\mathrm{CH}$ areas vary with the solar rotation, some data aggregation is required to reduce this effect. We replace total $\mathrm{CH}$ area for the current day with the maximal $\mathrm{CH}$ area within the 13-day window centred at the current day. Note that 13-day window roughly corresponds to a half of the solar rotation period. The same procedure is applied for SW speed data as well. The result is shown in the Fig. 11. Additional 150-day moving average, also shown in the Fig. 11, reveals long-term variations of the obtained time-series.

We observe that $\mathrm{CHs}$ area demonstrates yearly variations, increasing trend during the declining phase of the solar cycle and is minimal during the maximum of the solar cycle. In our opinion, the nature of near-yearly variations can be in North-South asymmetry and variations of the solar D-angle, while increasing of CHs area in the minimum of the solar cycle is associated with polar magnetic field strengthening. Note that $\mathrm{CH}$ areas vary in about three times within the solar cycle. We also note a correlation between averaged $\mathrm{CH}$ areas and averaged SW speeds. Numerical value of this correlation is 0.7 . More detailed investigation of the coupling between CHs and SW can be found e.g. in Rotter et al. (2015) and de Toma (2011). 


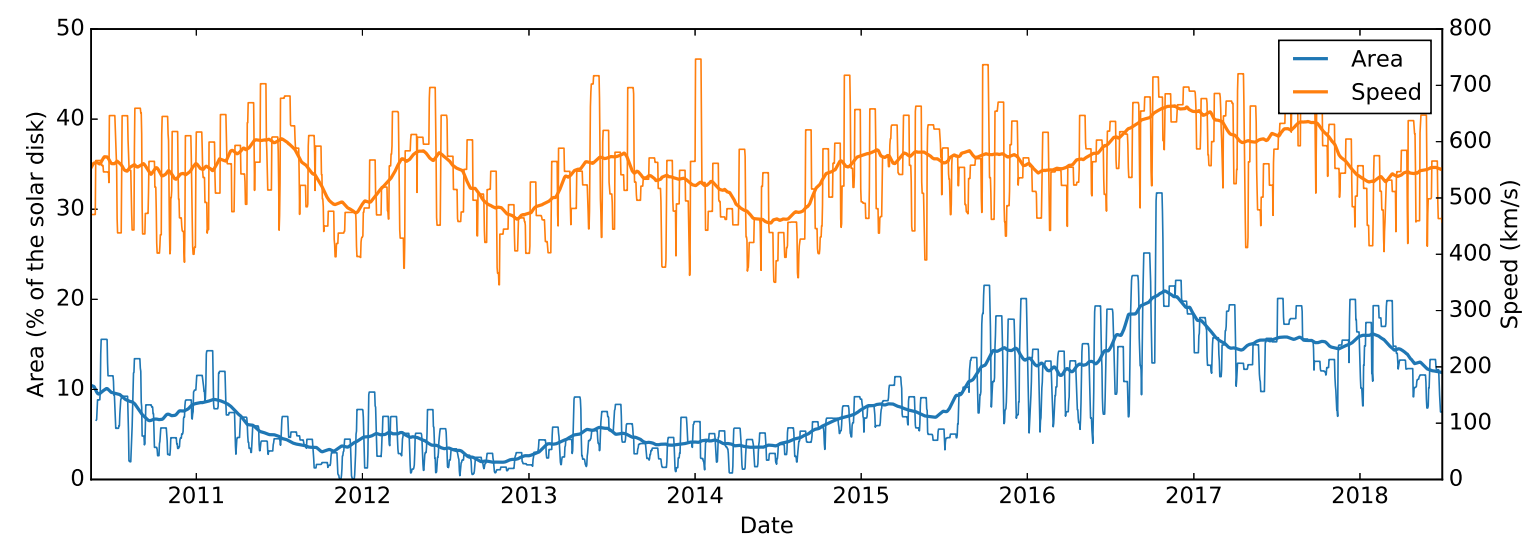

Figure 11. Maximal within 13-day period areas of coronal holes (blue line) and solar wind speed (orange line). Smoothed lines within each plot represent 150-day moving average.

\section{CONCLUSIONS}

Segmentation of coronal holes is a basic step in many space weather prediction models. However, as we have demonstrated, isolated $\mathrm{CHs}$ and their parameters vary dramatically from one algorithm to another one. In our opinion, the problem comes from the fact that hand-engineered algorithms do not have enough capacity to deal with a large variability of CHs. To overcome this limitation, we suggest an approach based on modern neural network architecture, known as U-Net, which has approved its effectiveness in various image segmentation problems.

We trained the U-Net neural network on a set of daily SDO/AIA $193 \AA$ solar disk images and corresponding $\mathrm{CH}$ segmentation maps for the time period from 2010 to 2017. Segmentation maps were provided by the Kislovodsk Mountain Astronomical Station. The time period from 2017 to June 2018 was used for model evaluation and comparison with other segmentation algorithms.

The source code that allows to reproduce the model architecture and training procedure is available at https://github.com/observethesun/coronal_holes.

Our first observation is that the trained neural network gives a better prediction of $\mathrm{CHs}$ in contrast to the semiautomatic procedure applied for $\mathrm{CH}$ segmentation in the training dataset. Thus, we conclude that the neural network is able to generalize and improve heuristics that were exploited for annotating of the training dataset.

Second, detailed comparison of $\mathrm{CHs}$ isolated by U-Net, SPOCA and CHIMERA algorithms shows that CHs isolated by U-Net correlate better with visual expectation from SDO/AIA $193 \AA$ A solar disk images. U-Net also provides better stability of segmentation maps in comparison to SPOCA.

Third, we observe very similar patterns in daily variation of total CHs area between all three algorithms, however, absolute values differ a lot.

We conclude that U-Net is able to give a reasonable segmentation of $\mathrm{CHs}$ in solar disk images. The most important advantage of this approach is that neural networks can learn and generalize a methodology of active region isolation even if this methodology is not formalized (e.g. in case of manual segmentation). Thus one can create extended homogeneous datasets of active regions.

As an example of homogeneous dataset we considered CHs isolated by U-Net from the beginning of SDO/AIA observations in 2010 (solar cycle 24). We observed that CHs area increases in about three times from minimal values in the maximum of the solar cycle to maximal values during the declining phase of the solar cycle. Comparison with the solar wind speed variations for the same period gives the correlation coefficient 0.7 .

We hope that this work will inspire further investigations of neural networks in application to analysis of solar images.

\section{ACKNOWLEDGEMENTS}

We are grateful to the referee for critical reading of the manuscripts. The research is supported by RSF under grant 15-12-20001 (AT) and RFBR under grant 18-02-00085 (EI).

\section{REFERENCES}

Abramenko V., Yurchyshyn V., Watanabe H., 2009, Solar Physics, 260, 43

Altschuler M. D., Trotter D. E., Orrall F. Q., 1972, Solar Physics, 26,354

Asensio Ramos A., Requerey I. S., Vitas N., 2017, A\&A, 604, A11

Barra, V. Delouille, V. Kretzschmar, M. Hochedez, J.-F. 2009, A\&A, 505, 361

Bishop C. M., 1995, Neural Networks for Pattern Recognition. Oxford University Press, Inc., New York, NY, USA

Chen L., Papandreou G., Kokkinos I., Murphy K., Yuille A. L., 2014, CoRR, abs/1412.7062

Ching T., et al., 2017, bioRxiv

Çiçek Ö., Abdulkadir A., Lienkamp S. S., Brox T., Ronneberger O., 2016, CoRR, abs/1606.06650

Clette F., Svalgaard L., Vaquero J. M., Cliver E. W., 2014, Space Science Reviews, 186, 35

Clevert D.-A., Unterthiner T., Hochreiter S., 2015, CoRR, abs/1511.07289

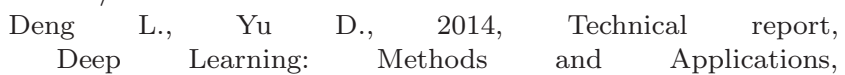


https://www.microsoft.com/en-us/research/publication/deep-learning-methods-and-applications/. https://www.microsoft.com/en-us/research/publication/deep-learning-methods-and-applications/

Díaz Baso C. J., Asensio Ramos A., 2018, A\&A, 614, A5

Dice L. R., 1945, Ecology, 26, 297

Dumoulin V., Visin F., 2016, A guide to convolution arithmetic for deep learning, http://arxiv.org/abs/1603.07285

Garton T. M., Gallagher P. T., Murray S. A., 2018, Journal of Space Weather and Space Climate, 8, A02

Goodfellow I., Bengio Y., Courville A., 2016, Deep Learning. MIT Press, http://www.deeplearningbook.org

Henney C. J., Harvey J. W., 2005, in Large-scale Structures and their Role in Solar Activity. p. 261 (arXiv: astro-ph/0701122)

Hong S., Kim J., Han J., Kim Y., 2014, in AGU Fall Meeting Abstracts. p. SH21A

Iglovikov V., Mushinskiy S., Osin V., 2017a, CoRR, abs/1706.06169

Iglovikov V., Rakhlin A., Kalinin A. A., Shvets A., 2017b, CoRR, abs/1712.05053

Kingma D. P., Ba J., 2014, CoRR, abs/1412.6980

Krieger A. S., Timothy A. F., Roelof E. C., 1973, Solar Physics, 29,505

Krista L. D., Gallagher P. T., 2009, Solar Physics, 256, 87

Lemen J. R., et al., 2012, Solar Physics, 275, 17

Meng Q., Chen W., Wang Y., Ma Z., Liu T.-Y., 2017, CoRR, abs/1709.10432

Nolte J. T., et al., 1976, Solar Physics, 46, 303

Obridko V. N., Shelting B. D., Livshits I. M., Asgarov A. B., 2009, Solar Physics, 260, 191

Perez L., Wang J., 2017, CoRR, abs/1712.04621

Priest E., 2014, Magnetohydrodynamics of the Sun. Cambridge University Press, https : //books.google.ru/books?id=BrbSAgAAQBAJ

Radiuk P. M., 2017, Information Technology and Management Science, 20, 20

Robbins S., Henney C. J., Harvey J. W., 2006, Solar Physics, 233, 265

Ronneberger O., P.Fischer Brox T., 2015, in Medical Image Computing and Computer-Assisted Intervention (MICCAI). Springer, pp 234-241, http://lmb. informatik. uni-freiburg.de/Publications/2015/RFB15a

Rotter T., Veronig A. M., Temmer M., Vršnak B., 2015, Solar Physics, 290, 1355

Scholl I. F., Habbal S. R., 2008, Solar Physics, 248, 425

Srivastava N., Hinton G., Krizhevsky A., Sutskever I., Salakhutdinov R., 2014, Journal of Machine Learning Research, 15, 1929

Stone E., Frandsen A., Mewaldt R., Christian E., Margolies D., Ormes J., Snow F., 1998, Space Science Reviews, 86, 1

Tlatov A., Tavastsherna K., Vasil'eva V., 2014, Solar Physics, 289,1349

Verbeeck, C. Delouille, V. Mampaey, B. De Visscher, R. 2014, A\&A, 561, A29

Zhang J., Woch J., Solanki S. K., von Steiger R., Forsyth R., 2003, Journal of Geophysical Research: Space Physics, 108

de Toma G., 2011, Solar Physics, 274, 195

This paper has been typeset from a $\mathrm{T}_{\mathrm{E}} \mathrm{X} / \mathrm{LAT} \mathrm{EX}$ file prepared by the author. 